\title{
ON SEQUENCES OF CONTINUOUS FUNCTIONS HAVING CONTINUOUS LIMITS*
}

BY

\author{
D. C. GILLESPIE AND W. A. HURWITZ
}

1. Introduction, notation and terminology. In order that the limit of a convergent sequence of continuous functions be a continuous function, it is sufficient, but not necessary, that the convergence be uniform; a necessary and sufficient condition is that the convergence be quasi-uniform. Consideration of the theory of summability leads to the observation that it is also sufficient that some sequence into which the given sequence can be transformed by a regular transformation should be uniformly convergent. It may happen that this condition is fulfilled when the original sequence converges non-uniformly. For example, let

$$
s_{n}(x)=\left\{\begin{array}{lr}
2^{n} x, & 0 \leqq x \leqq 1 / 2^{n} \\
2-2^{n} x, & 1 / 2^{n} \leqq x \leqq 1 / 2^{n-1} ; \\
0, & 1 / 2^{n-1} \leqq x \leqq 1
\end{array}\right.
$$

Then $s_{n}(x)$ is continuous, $0 \leqq x \leqq 1$. Furthermore, $s_{n}(x) \rightarrow 0$; for if $x=0$, $s_{n}(x)=0$ for all $n$, and if $0<x \leqq 1, s_{n}(x)=0$ when $n>1-(\log x) /(\log 2)$. The convergence is non-uniform, since $s_{n}\left(1 / 2^{n}\right)=1$. For the first Cesàro mean,

we have

$$
\sigma_{n}(x)=\frac{1}{n}\left\{s_{1}(x)+s_{2}(x)+\cdots+s_{n}(x)\right\},
$$

It follows that

$$
\sigma_{n}(x)= \begin{cases}\frac{1}{n}\left(2^{n+1}-2\right) x, & 0 \leqq x \leqq 1 / 2^{n} ; \\ \frac{1}{n}(2-2 x), & 1 / 2^{n} \leqq x \leqq 1 .\end{cases}
$$

and $\sigma_{n} \rightarrow 0$ uniformly.

$$
0 \leqq \sigma_{n}(x) \leqq \frac{2}{n}, \quad 0 \leqq x \leqq 1,
$$

It is natural to inquire whether every sequence $\left\{s_{n}(x)\right\}$, bounded for all $n$ and $x$, having its elements continuous, and converging to a continuous limit, can be transformed by an appropriately chosen regular transformation

* Presented to the Society, December 27, 1929; received by the editors November 22, 1929. 
into a uniformly convergent sequence; the present paper gives an affirmative answer to this question.* The primary object was to establish the result for a bounded closed interval of ordinary one-dimensional euclidean space; but in the course of the proof it became necessary to replace the given interval by a closed subset, so that nothing was lost by starting at once with any bounded closed set. Cast in this form, the work was seen to apply to sets in an abstract metric space; we therefore give all our results in this form. The reader not interested in the general case will find that every step has its usual meaning in a euclidean space of one or more dimensions if the term compact is interpreted as bounded.

Throughout, we denote by $x$ a point of a metric space. We write $A B$ for the greatest common subset, $A+B$ for the union, of two point sets $A$ and $B, A$ for the complement (in the whole space) of $A$, and $C$ or $\supset$ for the relation of inclusion. If $A$ is a compact closed set and $f(x)$ a function continuous in $A$, then $f(x)$ possesses a maximum in $A$, which we denote by $G(f ; A)$.

Let $S$ represent a sequence

$$
S: \quad s_{1}(x), s_{2}(x), s_{3}(x), \cdots
$$

of functions each continuous in $A$, such that, for some constant $M$,

$$
0 \leqq s_{n}(x) \leqq M,
$$

and such that

$$
\lim _{n \rightarrow \infty} s_{n}(x)=0 ;
$$

then we call $S$ an $S$-sequence in $A$.

2. The maximal function. Let $x$ be a point of $A$. For every sequence $\dagger$ $\left(x_{k}\right)$ of points of $A$ having $x$ as limit and every sequence $\left(n_{k}\right)$ of positive integers becoming infinite, form

$$
\limsup _{n \rightarrow \infty} s_{n_{k}}\left(x_{k}\right)=\lambda
$$

the least upper bound of all such numbers $\lambda$ is the value at $x$ of the maximal function $\ddagger$ of $S$ in $A$; it will be denoted by $H(S ; A ; x)$. At every point of $A$,

* It is evident that such a condition is more readily adapted to practical application than the A:zelà condition of quasi-uniform convergence. For instance, it shows immediately that the sequence of integrals of the elements of a sequence of the stated character is summable to the integral of the limit function.

$\dagger$ It is not assumed that $x_{k} \neq x$.

$\ddagger$ Essentially the same concept was used by Osgood, American Journal of Mathematics, vol. 19 (1897), p.166. Our formulation is identical with that of Hahn, Theorie der reellen Funktionen, 1921, p. 231. 
$H(S ; A ; x) \geqq 0$; at an isolated point of $A, H(S ; A ; x)=0$. For convenience we define $H(S ; A ; x)=0$ also at every point of $\bar{A}$.

I. If $S$ is an $S$-sequence in the compact closed set $A, H(S ; A ; x)$ is an upper semi-continuous function. If $B$ is a closed subset of $A$, then $H(S ; A ; x)$ $\geqq H(S ; B ; x)$.

The semi-continuity is a standard result.* The inequality follows at once from the definition. then

II. If $S$ is an S-sequence in a compact closed set $A, h>0$, and $H(S ; A ; x)<h$,

$$
\limsup _{n \rightarrow \infty} G\left(s_{n} ; A\right) \leqq h .
$$

Suppose it were true that

$$
\limsup _{n \rightarrow \infty} G\left(s_{n} ; A\right)>h .
$$

Then for a sequence of values $\left(n_{k}\right), G\left(s_{n_{k}} ; A\right)>h$. For each $k$ there is a point $x_{k}$ of $A$ for which $s_{n_{k}}\left(x_{k}\right)=G\left(s_{n_{k}} ; A\right)$; hence

$$
s_{n_{k}}\left(x_{k}\right)>h \text {. }
$$

The sequence of points $\left(x_{k}\right)$ has at least one limiting point $\xi$, which is a point of $A$. Let the notation be altered so that the indices $k$ refer only to a subsequence $\left(x_{k}\right)$ having $\xi$ as limit. Then (6) holds for all $k$, and

$$
\limsup _{k \rightarrow \infty} s_{n_{k}}\left(x_{k}\right) \geqq h \text {. }
$$

The left side of (7) is one of the values $\lambda$ of $(4)$; thus $H(S ; A ; \xi) \geqq h$, in contradiction to the hypothesis.

III. If $S$ is an $S$-sequence in a compact closed set $A$ and $h>0$, then the set $A^{1}$ of points at which $H(S ; A ; x) \geqq h$ is a closed proper subset of $A$, nowhere dense in $A$.

$A^{1}$ is closed $\dagger$ on account of the upper semi-continuity of $H(S ; A ; x)$. It is certainly a proper subset of $A$ if it is nowhere dense in $A$. To prove that $A^{1}$ is nowhere dense in $A$ we show that in each open set $C$ which contains a point of $A$ there is an open set which contains a point of $A$ and no point of $A^{1}$. Suppose this were untrue for a given open set $C$ containing a point $x_{0}$ of $A$. Then $C$ itself contains a point of $A^{1}$; that is, there is a point in $C$ for which $H \geqq h>h / 2$. Thus there is an index $n_{1}$ and a point $x_{1}$ of $A C$ such that

* Hahn, loc. cit., chapter IV, $\S 1$, Theorem IX.

† Hahn, loc. cit., chapter II, $\$ 9$, Theorem IV. 
$s_{n_{1}}\left(x_{1}\right)>h / 2$; and on account of the continuity of $s_{n_{1}}(x)$ there is an open set $C_{1}$, contained in $C$ and containing $x_{1}$, such that $s_{n_{1}}(x)>h / 2$ throughout $A C_{1}$. As $C_{1}$ contains a point of $A$, it contains a point of $A^{1}$, at which $H \geqq h>h / 2$; there is an index $n_{2}>n_{1}$ and a point $x_{2}$ of $A C_{1}$ such that $s_{n_{3}}\left(x_{2}\right)>h / 2$; and there is then an open set $C_{2}$, contained in $C_{1}$ and containing $x_{2}$, such that $s_{n_{1}}(x>h / 2)$ throughout $A C_{2}$. Continuing thus, we obtain a sequence of open sets, $C_{1}, C_{2}, C_{3}, \cdots$, each contained in the preceding, and such that $s_{n_{k}}\left(x_{k}\right)>h / 2$ throughout $A C_{k}$. Let $L_{k}$ denote the set obtained by adjoining to $A C_{k}$ its limit points. Then each of the sequence of closed sets $L_{1}, L_{2}, L_{3}, \cdots$ contains the following, and $s_{n_{k}}\left(x_{k}\right) \geqq h / 2$ throughout $L_{k}$. These sets have at least one point in common. At this point $\xi, s_{n_{k}}(\xi) \geqq h / 2$ for every $k$. If we let $k \rightarrow \infty$, we have $0 \geqq h / 2$, in contradiction to the hypothesis that $h>0$. We have thus shown that $A^{1}$ is nowhere dense in $A$.

IV. If $S$ is an $S$-sequence in a compact closed set $A, B$ a closed subset of $A$, and $h>0$, and if $A^{1}, B^{1}$ denote respectively the sets for which $B(S ; A ; x)$ $\geqq h, H(S ; B ; x) \geqq h$, then $B^{1} \subset A^{1}$.

This follows from $I$.

3. $T$-transformations. The transformations used to convert the sequence $S$ into a uniformly convergent sequence will be of a particular type, which we now discuss. Write the transformation

$$
T: \quad \sigma_{n}=\sum_{k=1}^{\infty} a_{n, k} s_{k},
$$

which carries the sequence $S$ into the sequence $\Sigma$ :

$$
\Sigma: \quad \sigma_{1}(x), \sigma_{2}(x), \sigma_{3}(x), \cdots
$$

in the abbreviated form $\Sigma=T(S)$. Such a transformation will be called a $\mathcal{T}$-transformation provided the following conditions are satisfied:

$$
a_{n, k} \geqq 0 ; \sum_{k=1}^{\infty} a_{n, k}=1 ;
$$

there exist integers $\mu_{n}, \nu_{n}$ such that

$$
\mu_{1} \leqq \nu_{1}<\mu_{2} \leqq \nu_{2}<\mu_{3} \leqq \nu_{3}<\cdots
$$

and that

$$
a_{n, k}=0 \text { unless } \mu_{n} \leqq k \leqq \nu_{n} .
$$

Obviously (12) makes it possible to write (8) in the form

$$
\sigma_{n}=\sum_{k=\mu_{n}}^{m_{n}} a_{n, k} s_{k}
$$


so that each row of the matrix $\left\|a_{n k}\right\|$ contains a finite set of non-zero elements and each column contains at most one such element.

It may be noted that the identity

$$
I: \quad \sigma_{n}=s_{n}
$$

is a T-transformation, and that the product of two $\mathcal{T}$-transformations is a T-transformation.

V. $A$ T-transformation is regular. Furthermore if $\Sigma=T(S)$, where $S$ is an S-sequence in the compact closed set $A$, then

$$
\lim _{n \rightarrow \infty} \sup G\left(\sigma_{n} ; A\right) \leqq \lim _{n \rightarrow \infty} \sup G\left(s_{n} ; A\right) \text {. }
$$

From (10), (11), (12) it is clear that the conditions for regularity are satisfied. To prove the second statement, write

$$
\limsup _{n \rightarrow \infty} G\left(s_{n} ; A\right)=l \text {. }
$$

For each $\epsilon>0$ there is an index $K$ such that when $k>K, G\left(s_{k} ; A\right)<l+\epsilon$.

Therefore when $n>N=\mu_{K}$,

$$
\sigma_{n} \leqq \sum_{k=\mu_{n}}^{n_{n}} a_{n, k} G\left(s_{k} ; A\right)<(l+\epsilon) \sum_{k=\mu_{n}}^{\nu_{n}} a_{n, k}=l+\epsilon
$$

throughout $A$, so that

$$
\limsup _{n \rightarrow \infty} G\left(\sigma_{n} ; A\right) \leqq l+\epsilon .
$$

Since this result holds for arbitrary $\epsilon>0$, it follows that

$$
\limsup _{n \rightarrow \infty} G\left(\sigma_{n} ; A\right) \leqq l .
$$

A $\mathcal{T}$-transformation, as defined, is not of the familiar form which possesses a triangular matrix; it is, however, equivalent to such a transformation. Let

$$
b_{n, k}=\left\{\begin{array}{l}
a_{m, k} \text { if } \nu_{m} \leqq n<\nu_{m+1}, \mu_{m} \leqq k \leqq \nu_{m} \\
0 \text { otherwise }
\end{array}\right.
$$

Then the transformation

$$
\tau_{n}=\sum_{k=1}^{n} b_{n, k} s_{k}
$$

has a triangular matrix, and is related to (8) as follows:

$$
\tau_{n}=\left\{\begin{array}{c}
0, n<\nu_{1} \\
\sigma_{m}, \nu_{m} \leqq n<\nu_{m+1} .
\end{array}\right.
$$


Thus the sequence $\left(\tau_{n}\right)$ is obtained (for $n \geqq \nu_{1}$ ) by appropriate repetition of the terms of $\left(\sigma_{n}\right)$. If either of these sequences converges, the other converges to the same value; if either converges uniformly, the other converges uniformly.

4. Application of $\tau$-transformations to $\delta$-sequences. We now prove several lemmas to facilitate the work of $\S \S 6,7$.

VI. Let $h \geqq 0$; let $S$ be an $S$-sequence in the compact closed set $A$. If for each $q>h$ there is a $\tau$-transformation $\Sigma^{(q)}=T_{q}(S)$ such that

$$
\limsup _{n \rightarrow \infty} G\left(\sigma_{n}^{(q)} ; A\right) \leqq q,
$$

then there is a $\mathcal{T}$-transformation $\Sigma=T(S)$ such that

$$
\limsup _{n \rightarrow \infty} G\left(\sigma_{n} ; A\right) \leqq h .
$$

Let $q, q_{1}, q_{2}, \cdots$ be a decreasing sequence of numbers having the limit $h$. Since lim sup $G\left(\sigma_{n}^{\left(q_{1}\right)} ; A\right) \leqq q_{1}<q$, we can choose $n_{1}$ so that $G\left(\sigma_{n_{1}}^{\left(q_{1}\right)} ; A\right)<q$. Again since $\lim \sup G\left(\sigma_{n}^{\left(q_{3}\right)} ; A\right) \leqq q_{2}<q_{1}$, it follows that for all sufficiently great $n, G\left(\sigma_{n}^{\left(q_{2}\right)} ; A\right)<q_{1}$. Choose $n_{2}$ so great as to satisfy this condition and also so great that $\sigma_{n_{2}}^{\left(q_{2}\right)}$ involves only elements of $S$ of greater subscript than those found in $\sigma_{n_{1}}^{\left(q_{1}\right)}$; this is possible since each $T_{q}$ is a $\tau$-transformation. Continuing thus, choose generally $\sigma_{n_{k}}^{\left(q_{k}\right)}$ so that

$$
G\left(\sigma_{n_{k}}^{\left(q_{k}\right)} ; A\right)<q_{k-1}
$$

and so that $\sigma_{n_{k}}^{\left(q_{k}\right)}$ contains only elements of $S$ of subscript greater than those found in $\sigma_{n_{k-1}}^{\left(q_{k-1}\right)}$. Now define

$$
T: \quad \sigma_{k}=\sigma_{n_{k}}^{\left(q_{k}\right)} .
$$

This is a $\mathcal{T}$-transformation. Also, by (19),

$$
G\left(\sigma_{k} ; A\right)<q_{k-1},
$$

and (18) follows at once.

In VI replace $h$ by $0, q$ by $h$; we state this special case:

VII. Let $S$ be an S-sequence in the compact closed set $A$. If for each $h>0$ there is a $\tau$-transformation $\Sigma^{(h)}=T_{h}(S)$ such that

$$
\limsup _{n \rightarrow \infty} G\left(\sigma_{n}^{(h)} ; A\right) \leqq h,
$$

then there is a $\tau$-transformation $\Sigma=T(S)$ such that $\sigma_{n} \rightarrow 0$ uniformly in $A$. 
VIII. Let $h>0$; let $S$ be an $S$-sequence in the compact closed set $A, B$ a closed subset of $A$. If

$$
\limsup _{n \rightarrow \infty} G\left(s_{n} ; B\right)<h,
$$

and if for each neighborhood ${ }^{*} C$ of $B$ in $A$ there exists a $\mathcal{T}$-transformation $\Sigma^{(C)}=T^{(C)}(S)$ such that

$$
\limsup _{n \rightarrow \infty} G\left(\sigma_{n}^{(C)} ; A \bar{C}\right)<h,
$$

then there exists a $\mathcal{T}$-transformation $\Sigma=T(S)$ such that

$$
\limsup _{n \rightarrow \infty} G\left(\sigma_{n} ; A\right) \leqq h \text {. }
$$

Denote an index $n$ chosen at random (for example, $n=1$ ) by $m_{1,1}$ and define

$$
\sigma_{1}=s_{m_{1,1}} .
$$

This will be the first formula in the definition of $T$.

On account of (20), for all sufficiently great $n, s_{n}<h$ throughout $B$. Choose $m_{2,1}>m_{1,1}$ so that $s_{m_{2,1}}<h$ throughout $B$. Since $s_{m_{2,1}}$ is continuous, there is a neighborhood $C_{2,1}$ of $B$ in $A$ such that $\dagger s_{m_{2,1}}<h$ throughout $C_{2,1}$. Form the transformation $T^{\left(C_{2,1}\right)}$; by $(21)$, for all sufficiently great $n, \sigma_{n}^{\left(C_{2,1}\right)}<h$ in $A \bar{C}_{2,1}$. Choose $m_{2,2}$ so that $\sigma_{m_{2,2}}^{\left(C_{2,1}\right)}<h$ in $A \bar{C}_{2,1}$, and so that $\sigma_{m_{2,2}}^{\left(C_{2,1}\right)}$ contains only elements of $S$ of subscript greater than $m_{1,1}$. Define

$$
\sigma_{2}=\frac{1}{2}\left\{s_{m_{2,1}}+\sigma_{m_{2,2}}{ }^{\left(C_{2,1}\right)}\right\} \text {. }
$$

We see that $\sigma_{2}$ involves elements of $S$ of a finite set of indices of which the least is greater than $m_{1,1}$.

We continue the definition by induction. Suppose that $\sigma_{1}, \sigma_{2}, \cdots, \sigma_{k-1}$ have been written so that each contains terms of $S$ having a finite set of indices, of which the least in any row is greater than the greatest in the preceding row; we show how to form $\sigma_{k}$. Call $\nu$ the greatest subscript of an element of $S$ appearing in $\sigma_{k-1}$. As before choose $m_{k, 1}>\nu$ and such that $s_{m_{k, 1}}<h$ throughout $B$; then choose a neighborhood $C_{k, 1}$ of $B$ in $A$ such that $s_{m_{k, 1}}<h$ throughout $C_{k, 1}$. Form $T^{\left(C_{k, 1}\right)}$; choose $m_{k, 2}>\nu$ so that $\sigma_{m_{k, 2}}^{\left(C_{k, 1}\right)}<h$ in $A \bar{C}_{k, 1}$ and also $\sigma_{m_{k, 2}}^{\left(C_{k, 1}\right)}<h$ in $B$; then choose a neighborhood $C_{k, 2}$ of $B$ in $C_{k, 1}$ such that $\sigma_{m_{k, 2}}^{\left(C_{k, 1}\right)}<h$ in $C_{k, 2}$. Next form $T^{\left(C_{k, 2}\right)}$; choose $m_{k, 3}>\nu$ so that

* By a neighborhood of $B$ in $A$ we mean a subset of $A$ open in $A$ and containing $B$.

$\dagger$ For each point $b$ in $B$ a neighborhood of $b$ in $A$ exists throughout which $s_{m_{2,1}}<h$; the union of all such point-neighborhoods will be a possible $C_{2,1}$. 
$\sigma_{m_{k, 3}}^{\left(C_{h, 2}\right)}<h$ in $A \bar{C}_{k, 2}$ and also $\sigma_{m_{k, 3}}^{\left(C_{k, 2}\right)}<h$ in $B$; then choose a neighborhood $C_{k, 3}$ of $B$ in $C_{k, 2}$ such that $\sigma_{m, 2}^{\left(C_{k, 2}\right)}<h$ in $C_{k, 8}$. Continue thus, choosing the indices, neighborhoods, and transformations:

$$
\begin{gathered}
m_{k, 1}, C_{k, 1}, T^{\left(C_{k, 1}\right)} ; m_{k, 2}, C_{k, 2}, T^{\left(C_{k, 2}\right)} ; \cdots ; \\
m_{k, k-1}, C_{k, k-1}, T^{\left(C_{k, k-1}\right)} ; m_{k, k} ;
\end{gathered}
$$

so that

$$
\begin{gathered}
A \supset C_{k, 1} \supset C_{k, 2} \supset \cdots \supset C_{k, k-1} ; \\
\left\{\begin{aligned}
s_{m_{k, 1}}<h \text { in } C_{k, 1} ; & (p=1,2, \cdots, k-1) ; \\
\sigma_{m_{k, p+1}}^{\left(C_{k, p}\right)}<h \text { in } A \bar{C}_{k, p} & (p=1,2, \cdots, k-2) . \\
\sigma_{m_{k, p+1}}^{\left(C_{k, p}\right)}<h \text { in } C_{k, p+1} &
\end{aligned}\right.
\end{gathered}
$$

The set $A$ can be decomposed into the following parts having no points in common:

$$
\boldsymbol{H}=A \bar{C}_{k, 1}+C_{k, 1} \bar{C}_{k, 2}+C_{k, 2} \bar{C}_{k, 3}+\cdots+C_{k, k-2} \bar{C}_{k, k-1}+C_{k, k-1} .
$$

In $A \bar{C}_{k, 1}$ (which is contained in $A \bar{C}_{k, p}$ for $p \leqq k-1$ ), by (2) and (26),

$$
s_{m k, 1} \leqq M ; \quad \sigma_{m_{k, p}}^{\left(C_{k, p-1}\right)}<h \text { for } p>1 .
$$

In $C_{k, r-1} \bar{C}_{k, r}$ (which is contained in $A \bar{C}_{k, p}$ for $p \geqq r$ and in $C_{k, p}$ for $p \leqq r-1$ ), by (2), (26), and (10),

$$
s_{m_{k, 1}}<h ; \quad \sigma_{m_{k, p}}^{\left(C_{k, p-1}\right)}<h \text { for } p \neq r ; \quad \sigma_{m_{k, r}}^{\left(C_{k, r-1}\right)} \leqq M .
$$

In $C_{k, k-1}$ (which is contained in $C_{k, p}$ for $p \leqq k-1$ ),

$$
s_{m_{k, 1}}<h ; \quad \sigma_{m_{k, p}}^{\left(C_{k, p-1}\right)}<h \text { for } p \neq k ; \sigma_{m_{k, k}}^{\left(C_{k, k-1}\right)} \leqq M .
$$

Thus throughout $A$

$$
s_{m_{k, 1}}+\sigma_{m_{k, 2}}^{\left(C_{k, 1}\right)}+\sigma_{m_{k, 3}}^{\left(C_{k, 2}\right)}+\cdots+\sigma_{m_{k, k}}^{\left(C_{k, k-1}\right)}<(k-1) h+M .
$$

We now define the $k$ th row of the transformation $T$ as follows:

$$
\sigma_{k}=\frac{1}{k}\left\{s_{m_{k, 1}}+\sigma_{m_{k, 2}}^{\left(c_{k, 1}\right)}+\sigma_{m_{k, 3}}^{\left(C_{k, 2}\right)}+\cdots+\sigma_{m_{k, k}}^{\left(c_{k, k-1}\right)}\right\} .
$$

The transformation which has thus been defined is a $\mathfrak{T}$-transformation, since (10), (11), (12) are easily seen to hold. Also, from (27) and (28) we have throughout $A$ 


$$
\sigma_{k}<\frac{1}{k}\{(k-1) h+M\}=h+\frac{M-h}{k} ;
$$

thus

$$
G\left(\sigma_{n} ; A\right)<h+\frac{M-h}{n},
$$

and (22) follows at once.

IX. Let $h>0$; let $S$ be an $S$-sequence in the compact closed set $A, B$ a closed subset of $A$. If

$$
\limsup _{n \rightarrow \infty} G\left(s_{n} ; B\right) \leqq h
$$

and if for each neighborhood $C$ of $B$ in $A$ there is a $\tau$-transformation $\Sigma^{(C)}=$ $T^{(C)}(S)$ such that

$$
\limsup _{n \rightarrow \infty} G\left(\sigma_{n}{ }^{(C)} ; A \bar{C}\right) \leqq h,
$$

then there exists a $\mathcal{T}$-transformation $\Sigma=T(S)$ such that

$$
\limsup _{n \rightarrow \infty} G\left(\sigma_{n} ; A\right) \leqq h .
$$

Let $q>h$. Then $q$ satisfies the conditions imposed on $h$ in VIII; thus for

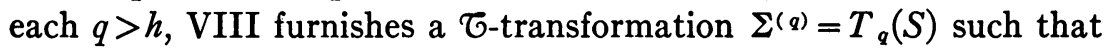

$$
\limsup _{n \rightarrow \infty} G\left(\sigma_{n}^{(q)} ; A\right) \leqq q .
$$

The conclusion of the present theorem then follows from VI.

5. Definition and properties of $h$-sets and $h$-order. Let $S$ be an $S$-sequence in the compact closed set $A$ and let $h>0$. We define a set of sets $A^{\alpha}$, where $\alpha$ is any Cantor ordinal of the first or second class, by the following scheme of induction:

(1) $A^{0}=A$;

(2) if $\alpha$ is not a limiting ordinal, then $A^{\alpha}$ is the set of points at which $H\left(S ; A^{\alpha-1} ; x\right) \geqq h$;

(3) if $\alpha$ is a limiting ordinal, then $A^{\alpha}$ is the greatest common subset of all $A^{\rho}$ for $\rho<\alpha$.

The sets thus defined we call the $h$-sets generated by $(S ; A)$. By the process of definition the set of $h$-sets is normally ordered. Several properties will be noted.

X. If $B$ is a closed subset of $A$ and if $B^{\alpha}$ denote the h-sets for $(S ; B)$, then $B^{\alpha} \subset A^{\alpha}$. 
The statement holds, by the hypothesis, when $\alpha=0$. We show that if it holds for each ordinal $\rho<\alpha$, it holds also for $\alpha$. Suppose that whenever $\rho<\alpha, B^{\rho} \subset A^{\rho}$. If $\alpha$ is not a limiting ordinal, $B^{\alpha-1} \subset A^{\alpha-1}$, and by IV, $B^{\alpha} \subset A^{\alpha}$. If $\alpha$ is a limiting ordinal, $B^{\alpha}$ is the greatest common subset of all $B^{p}$ for $\rho<\alpha$; each such $B^{\rho} \subset A^{\rho}$; hence $B^{\alpha}$ is contained in the greatest common subset of all $A^{\rho}$ for $\rho<\alpha$, which is $A^{\alpha}$.

XI. For $(S ; A)$ each $h$-set is a closed subset of each preceding $h$-set; each $h$-set is a proper subset of each preceding non-empty $h$-set.

That $A^{1}$ is a closed proper subset of $A^{0}$ is stated in III. Suppose that for $\rho<\alpha, A^{\rho}$ is a closed subset of every earlier $h$-set and a proper subset of each earlier non-empty $h$-set. If $\alpha$ is not a limiting ordinal it follows from III, with $A$ replaced by $A^{\alpha-1}$, that $A^{\alpha}$ is a closed proper subset of $A^{\alpha-1}$ (and hence of all earlier $h$-sets) unless $A^{\alpha-1}$ is empty; if $A^{\alpha-1}$ is empty, $A^{\alpha}$ will also be empty, since $H\left(S ; A^{\alpha-1} ; x\right)$ will then be zero throughout the space. If $\alpha$ is a limiting ordinal, and no preceding $h$-set is empty, let $\alpha_{n} \rightarrow \alpha$; then $A^{\alpha}$ is the greatest common subset of all $A^{\alpha_{n}}$, and any ordinal $\beta<\alpha$ is followed by some $\alpha_{n}$, so that $A^{\alpha}$ is a subset of $A^{\alpha_{n}}$, which is a proper subset of $A^{\beta}$; if $\alpha$ is a limiting ordinal and some preceding $h$-set is empty, $A^{\alpha}$ is empty.

XII. For $(S ; A)$ there is a non-limiting ordinal $\gamma$ such that $A^{\gamma}$ and all following $h$-sets are empty, while all preceding $h$-sets are non-empty.

If $A^{\alpha}$ were non-empty for every $\alpha$, the set of $h$-sets would constitute a normally ordered set of closed sets such that each is a proper subset of all its predecessors; such a set of sets must be countable; ${ }^{*}$ but the set of ordinals of first and second classes is not countable, $\dagger$ so that we should have a contradiction. Let $\gamma$ be the first ordinal for which $A^{\gamma}$ is empty; then $\gamma$ is not a limiting ordinal. For if $\gamma$ were a limiting ordinal, let $\gamma_{n} \rightarrow \gamma$; then $A^{\gamma}$ would be the greatest common subset of the descending sequence of non-empty closed sets $A^{\gamma_{n}}$ and hence could not be empty. $\ddagger$

Since $\gamma$, as given by XII, is not a limiting ordinal, we may write $\alpha=\gamma-1$. Then $A^{\alpha}$ and all preceding $h$-sets are non-empty, while all following $h$-sets are empty. We define the $h$-order of $(S ; A)$ as the greatest ordinal $\alpha$ for which $A^{\alpha}$ is not empty.

XIII. If $\alpha$ is the h-order of $(S ; A)$ then

$$
\limsup _{n \rightarrow \infty} G\left(s_{n} ; A^{\alpha}\right) \leqq h \text {. }
$$

\footnotetext{
* Hahn, loc. cit., chapter I, §7, Theorem XII.

$\dagger$ Hahn, loc. cit., Introduction, \$4, Theorem XIV.

$\ddagger$ Hahn, loc. cit., chapter I, §2, Theorem VIII.
} 
Since $A^{\alpha+1}$ (which is the set of points at which $H\left(S ; A^{\alpha} ; x\right) \geqq h$ ) is empty, $H\left(S ; A^{\alpha} ; x\right)<h$; the conclusion follows from II with $A$ replaced by $A^{\alpha}$.

6. Transformation of an $S$-sequence into a uniformly convergent sequence. In this section we prove two theorems which imply the principal result given in the next section.

$\mathrm{XIV}$. Let $h>0$. If $S$ is an $S$-sequence in the compact closed set $A$, there exists a $\mathcal{T}$-transformation $\Sigma=T(S)$ such that

$$
\limsup _{n \rightarrow \infty} G\left(\sigma_{n} ; A\right) \leqq h .
$$

We prove this by ordinal induction on $\alpha$, the $h$-order of $(S ; A)$. If $\alpha=0$, then (29) holds, by XIII, since the identity is a $\mathcal{T}$-transformation. It will be shown that if the conclusion holds for every closed compact set $Q$ such that the $h$-order of $(S ; Q)$ is less than $\alpha$, it holds for the set $A$ such that the $h$-order of $(S ; A)$ is $\alpha$.

Let $C$ be a neighborhood of $A^{\alpha}$ in $A$. Since $A^{\alpha} \subset C, A^{\alpha} \bar{C}$ is empty. Form $Q=A \bar{C}$ and let $Q^{\rho}$ represent the $h$-sets generated by $(S ; Q)$. Then $Q^{\rho} \subset A^{\rho}$, by $\mathrm{X}$, and $Q^{\rho} \subset Q \subset \bar{C}$; hence $Q^{\rho} \subset A^{\rho} \bar{C}$. Thus $Q^{\alpha}$ is empty, and the $h$-order of $(S ; Q)$ is some ordinal $\beta<\alpha$. The hypothesis of our theorem must then hold with $A$ replaced by $Q$ and $\alpha$ by $\beta$; the conclusion yields a $\mathcal{\tau}$-transformation $\Sigma^{\prime}=T^{\prime}(S)$ such that

$$
\limsup _{n \rightarrow \infty} G\left(\sigma_{n}^{\prime} ; Q\right) \leqq h .
$$

The transformation thus obtained depends on $Q$, and hence on the choice of $C$; indicating this by proper notation and replacing $Q$ by $A \bar{C}$, we see that for each neighborhood $C$ of $A^{\alpha}$ in $A$ we have found a $\mathcal{T}$-transformation $\Sigma^{(C)}=T^{(C)}(S)$ such that

$$
\limsup _{n \rightarrow \infty} G\left(\sigma_{n}{ }^{(C)} ; A \bar{C}\right) \leqq h
$$

But by XIII,

$$
\limsup _{n \rightarrow \infty} G\left(s_{n} ; A^{\alpha}\right) \leqq h .
$$

The hypotheses of IX are then satisfied with $B$ replaced by $A^{\alpha}$; the conclusion of IX furnishes the conclusion of our theorem.

$\mathrm{XV}$. Let $S$ be an $\mathrm{S}$-sequence in the compact closed set $A$. Then there exists $a$ T-transformation $\Sigma=T(S)$ such that $\sigma_{n} \rightarrow 0$ uniformly in $A$.

For each $h>0$ there exists by XIV a $\mathcal{T}$-transformation $\Sigma^{(h)}=T_{h}(S)$ such that 


$$
\underset{n \rightarrow \infty}{\lim \sup } G\left(\sigma_{n}^{(h)} ; A\right) \leqq h ;
$$

the theorem follows from VII.

7. The principal theorem. We easily deduce from XV the result mentioned in $\$ 1$.

XVI. Let $A$ be a compact closed set; let the sequence

$$
s_{1}(x), s_{2}(x), s_{3}(x), \cdots
$$

have the following properties:

(i) for each $n, s_{n}(x)$ is continuous in $A$;

(ii) for each $x$ in $A, s_{n}(x) \rightarrow s(x)$;

(iii) $s(x)$ is continuous in $A$;

(iv) there is a constant $M$ such that for all $x$ in $A$ and all $n,\left|s_{n}(x)\right| \leqq M$. Then there exists a $\mathcal{T}$-transformation $\Sigma=T(S)$ such that $\sigma_{n}(x) \rightarrow s(x)$ uniformly in $A$.

For all $x$ in $A,|s(x)| \leqq M$, by (i), (iv), and hence $\left|s_{n}(x)-s(x)\right| \leqq 2 M$. Call $s_{n}^{\prime}(x)=\left|s_{n}(x)-s(x)\right|$. Then

$$
S^{\prime}: \quad s_{1}^{\prime}(x), s_{2}^{\prime}(x), s_{3}^{\prime}(x), \cdots
$$

is an $S$-sequence in $A$. By XV there is a $\mathcal{T}$-transformation which, expressed in terms of $S^{\prime}$, gives $\Sigma^{\prime}=T\left(S^{\prime}\right)$ such that $\sigma_{n}^{\prime} \rightarrow 0$ uniformly in $A$. Let $\Sigma=T(S)$. Since no coefficient in a $\mathcal{T}$-transformation is negative and the sum of the coefficients in any row is 1 ,

$$
\begin{aligned}
\left|\sigma_{n}-s\right| & =\left|\left(\Sigma a_{n, k} s_{k}\right)-s\right|=\left|\Sigma a_{n, k}\left(s_{k}-s\right)\right| \\
& \leqq \Sigma a_{n, k}\left|s_{k}-s\right|=\Sigma a_{n, k} s_{k}^{\prime}=\sigma_{n}^{\prime},
\end{aligned}
$$

and $\sigma_{n} \rightarrow s$ uniformly in $A$.

8. Limitation on extension of results. As has been seen, a necessary and sufficient condition that a sequence satisfying (i), (ii), and (iv) of XVI have a continuous limit is that it be uniformly summable by a properly chosen regular transformation. The range of applicability of this result differs from the range of the Arzelà condition only by the assumption of condition (iv). How far, if at all, this condition may be relaxed, remains an open question; it is at any rate possible to show that too rapid increase in the violence of oscillation of the elements of the sequence may entirely prevent uniform summability.

Denote by $J$ the interval $0 \leqq x \leqq 1$ and by $J_{k}$ the interval $1 / 2^{k} \leqq x \leqq 1 / 2^{k-1}$. For each $n$ let* $s_{n}(x)=0$ in $J$ except in $J_{n}$; let $s_{n}(x)=0$ at the ends of $J_{n}$,

* For example, $s_{n}$ might be defined by an isosceles triangle of height $M_{n}$ over $J_{n}$ and have the value 0 elsewhere in $J$. 
have a maximum $M_{n} \geqq M>0$ at a point $x_{n}$ of $J_{n}$, and be continuous and non-negative in $J_{n}$. It is clear that $s_{n}(x) \rightarrow 0$ non-uniformly in $J$. We show further:

If the series

$$
\frac{1}{M_{1}}+\frac{1}{M_{2}}+\frac{1}{M_{3}}+\cdots
$$

converges, then there is no regular transformation of the form

$$
\sigma_{n}(x)=\sum_{k=1}^{\infty} a_{n, k} s_{k}(x)
$$

such that $\sigma_{n}(x) \rightarrow 0$ uniformly in $J$.

Call $R$ the value of the series (30). We have $\sigma_{n}(x)=a_{n, k} s_{k}(x)$ in $J_{k}$. If $\sigma_{n}(x) \rightarrow 0$ uniformly in $J$, then for each $\epsilon>0$ there exists $N$ independent of $x$ such that when $n>N,\left|\sigma_{n}(x)\right|<\epsilon$ throughout $J$. Thus for $n>N$, $\left|a_{n, k}\right| s_{k}(x)<\epsilon$ in $J_{k}$. In particular (since $x_{k}$ is in $J_{k}$ ) for $n>N,\left|a_{n, k}\right| M_{k}<\epsilon$; that is

$$
\left|a_{n, k}\right|<\frac{\epsilon}{M_{k}} \text { for } n>N ; \quad k=1,2,3, \cdots .
$$

Hence for $n>N$,

Therefore.

$$
\left|\sum_{k=1}^{\infty} a_{n, k}\right| \leqq \sum_{k=1}^{\infty}\left|a_{n, k}\right|<\sum_{k=1}^{\infty} \frac{\epsilon}{M_{k}}=R \epsilon .
$$

$$
\lim _{n \rightarrow \infty} \sum_{k=1}^{\infty} a_{n, k}=0 .
$$

But for any regular transformation this limit must be 1 , and we have a contradiction.

The $\tau$-transformations which we have used in this paper form a class obviously well adapted to the proof of the theorem. It might be asked whether some more special class of transformations of familiar type could be used for the same purpose. Some light is thrown on this question by a simple example of an $\delta$-sequence which cannot be transformed into a uniformly convergent sequence by any Cesàro mean. Let

$$
s_{n}(x)= \begin{cases}n x, & 0 \leqq x \leqq 1 / n \\ n-n(n-1) x, & 1 / n \leqq x \leqq 1 /(n-1) ; \\ 0, & 1 /(n-1) \leqq x \leqq 1\end{cases}
$$

It is clear that $s_{n}(x)$ is continuous and has the limit 0 in the interval $0 \leqq x \leqq 1$, 
and that $0 \leqq s_{n}(x) \leqq 1$. Form the Cesàro mean of order $r$ :

$$
\sigma_{n}(x)=\frac{\sum_{k=1}^{n}\left(\begin{array}{c}
n-k+r-1 \\
r-1
\end{array}\right) s_{k}(x)}{\left(\begin{array}{c}
n+r-1 \\
r
\end{array}\right)} .
$$

If $\sigma_{n}(x) \rightarrow 0$ uniformly, then in particular, $\sigma_{n}(1 / n) \rightarrow 0$. Since $s_{k}(1 / n)=k / n$ when $k \leqq n$,

$$
\sigma_{n}\left(\frac{1}{n}\right)=\frac{\sum_{k=1}^{n}\left(\begin{array}{c}
n-k+r-1 \\
r-1
\end{array}\right) \frac{k}{n}}{\left(\begin{array}{c}
n+r-1 \\
r
\end{array}\right)} .
$$

To evaluate the numerator, we note that the power series

$$
\begin{aligned}
& \sum_{k=1}^{\infty} k t^{k}=t(1-t)^{-2}, \\
& \sum_{p=0}^{\infty}\left(\begin{array}{c}
r+p-1 \\
r-1
\end{array}\right) t^{p}=(1-t)^{-r}, \\
& \sum_{n=1}^{\infty}\left(\begin{array}{c}
n+r \\
r+1
\end{array}\right) t^{n}=t(1-t)^{-r-2}
\end{aligned}
$$

are all absolutely convergent for $|t|<1$. If we compare the coefficient of $t^{n}$ in the Cauchy product of (32), (33) with that in (34), we see that

$$
\sum_{k=1}^{n} k\left(\begin{array}{c}
n-k+r-1 \\
r-1
\end{array}\right)=\left(\begin{array}{c}
n+r \\
r+1
\end{array}\right) \text {. }
$$

Hence, by (31),

and

$$
\sigma_{n}\left(\frac{1}{n}\right)=\frac{\frac{1}{n}\left(\begin{array}{c}
n+r \\
r+1
\end{array}\right)}{\left(\begin{array}{c}
n+r-1 \\
r
\end{array}\right)}=\frac{n+r}{n} \frac{1}{r+1}
$$

$$
\lim _{n \rightarrow \infty} \sigma_{n}\left(\frac{1}{n}\right)=\frac{1}{r+1}>0
$$

It is well known that the Euler-Abel power series transformation of a sequence into a function includes all the Cesâro means. Since the limit (35) is more nearly zero the higher the order $r$, it might be hoped that the Euler- 
Abel transformation would yield uniformity. However, this is not the case. Write

If

$$
\sigma(x, t)=(1-t) \sum_{k=1}^{\infty} s_{k}(x) t^{k-1} .
$$

$$
\lim _{t \rightarrow 1-} \sigma(x, t)=0
$$

uniformly for all $x$ in the interval, then in particular

$$
\lim _{n \rightarrow \infty} \sigma\left(\frac{1}{n}, 1-\frac{1}{n}\right)=0 \text {. }
$$

But

$$
\begin{aligned}
\sigma\left(\frac{1}{n}, t\right) & =(1-t) \sum_{k=1}^{n} \frac{k}{n} t^{k-1} \\
& =\frac{1-t}{n} \frac{d}{d t} \sum_{k=0}^{n} t^{k} \\
& =\frac{1-t}{n} \frac{d}{d t} \frac{1-t^{n+1}}{1-t} \\
& =\frac{1+t^{n}(n t-n-1)}{n(1-t)}
\end{aligned}
$$

therefore

$$
\sigma\left(\frac{1}{n}, 1-\frac{1}{n}\right)=1-2\left(1-\frac{1}{n}\right)^{n}
$$

and

$$
\lim _{n \rightarrow \infty} \sigma\left(\frac{1}{n}, 1-\frac{1}{n}\right)=1-\frac{2}{e}>0 .
$$

The Borel transformation also fails to bring about uniform convergence. Write

If

$$
\sigma(x, t)=e^{-t} \sum_{k=1}^{\infty} s_{k}(x) \frac{t^{k-1}}{(k-1) !} .
$$

$$
\lim _{t \rightarrow+\infty} \sigma(x, t)=0
$$

uniformly for all $x$ in the interval, then in particular

$$
\lim _{n \rightarrow \infty} \sigma\left(\frac{1}{n}, n\right)=0 \text {. }
$$

We shall prove that, instead, 


$$
\lim _{n \rightarrow \infty} \sigma\left(\frac{1}{n}, n\right)=\frac{1}{2} \text {. }
$$

We have

$$
\begin{aligned}
\sigma\left(\frac{1}{n}, t\right) & =e^{-t} \sum_{k=1}^{n} \frac{k}{n} \frac{t^{k-1}}{(k-1) !} \\
& =e^{-t} \sum_{k=1}^{n} \frac{k-1}{n} \frac{t^{k-1}}{(k-1) !}+e^{-t} \sum_{k=1}^{n} \frac{1}{n} \frac{t^{k-1}}{(k-1) !} \\
& =e^{-t} \sum_{k=1}^{n-1} \frac{1}{n} \frac{t^{k}}{(k-1) !}+e^{-t} \sum_{k=1}^{n} \frac{1}{n} \frac{t^{k-1}}{(k-1) !} \\
& =\frac{t+1}{n} e^{-t} \sum_{k=1}^{n} \frac{t^{k-1}}{(k-1) !}-\frac{e^{-t} t^{n}}{n !}
\end{aligned}
$$

hence

$$
\sigma\left(\frac{1}{n}, n\right)=\left(1+\frac{1}{n}\right) e^{-n} \sum_{k=1}^{n} \frac{n^{k-1}}{(k-1) !}-\frac{e^{-n} n^{n}}{n !} .
$$

By Stirling's theorem, the second term on the right has the limit zero. It thus.suffices to show that

$$
\lim _{n \rightarrow \infty} e^{-n} \sum_{k=1}^{n} \frac{n^{k-1}}{(k-1) !}=\frac{1}{2}
$$

or that

$$
\lim _{n \rightarrow \infty} e^{-n} \sum_{k=1}^{n+1} \frac{n^{k-1}}{(k-1) !}=\frac{1}{2},
$$

since the added term, again by Stirling's theorem, has the limit zero. From the identity

$$
e^{-z} \frac{z^{n}}{n !}=\frac{d}{d z}\left\{-e^{-z} \sum_{k=1}^{n+1} \frac{z^{k-1}}{(k-1) !}\right\}
$$

we find by integration from 0 to $n$ that

$$
\int_{0}^{n} e^{-z} \frac{z^{n}}{n !} d z=1-e^{-n} \sum_{k=1}^{n+1} \frac{n^{k-1}}{(k-1) !}
$$

thus (37) is equivalent to

$$
\lim _{n \rightarrow \infty} \int_{0}^{n} e^{-z} \frac{z^{n}}{n !} d z=\frac{1}{2}
$$


This is a standard result.*

It can also be shown that no linear transformation with triangular matrix which is totally regular and permutable with the first Cesàro mean can yield in the present example a uniformly convergent sequence.

Nevertheless, so simple a totally regular transformation as the following will suffice:

$$
\sigma_{n}(x)=\frac{\frac{s_{1}(x)}{1}+\frac{s_{2}(x)}{2}+\cdots+\frac{s_{n}(x)}{n}}{1+\frac{1}{2}+\cdots+\frac{1}{n}} .
$$

In fact, denoting by $D_{n}$ the denominator of (38), we have

Thus

$$
\sigma_{n}(x)= \begin{cases}n x / D_{n}, & 0 \leqq x \leqq 1 / n \\ 1 / D_{n}, & 1 / n \leqq x \leqq 1\end{cases}
$$

$$
0 \leqq \sigma_{n}(x) \leqq \frac{1}{D_{n}}, 0 \leqq x \leqq 1 ;
$$

and $\sigma_{n}(x) \rightarrow 0$ uniformly.

* For instance it is a special case of the formula given by Pólya-Szegö, Aufgaben und Lehrsutze aus der Analysis, vol. I, Part 2, Problem 210, with $\alpha=\beta=0$.

Cornell UNIVERSITY,

ITHACA, N. Y. 